\title{
Alterations in the Thickness of Motor Cortical Subregions After Motor-Skill Learning and Exercise
}

\author{
Brenda J. Anderson, ${ }^{1,2,4}$ Paul B. Eckburg, ${ }^{3}$ and Karen I. Relucio ${ }^{3}$ \\ ${ }^{1}$ Department of Psychology, ${ }^{2}$ Program in Neurobiology, State University of New York at Stony Brook, Stony Brook, New York 11794-2500, USA; \\ ${ }^{3}$ Stanford University Medical School, Stanford, California 94305, USA
}

\begin{abstract}
Behavioral manipulations such as housing in an enriched environment have been shown to increase brain weight and visual cortical thickness. The present study was designed to test whether skill learning or repetitive movements can alter the thickness of the motor cortex. One group of 6-mo-old Long-Evans female rats learned motor skills on an obstacle course that increased in difficulty over training and required balance and coordination. A second group ran voluntarily in exercise wheels attached to their home cage but had little opportunity for skill learning. The third group was handled daily but received no opportunity for learning or exercise. Each condition lasted 26-29 d. The skill-learning and exercise conditions had greater heart weight, and the exercise condition had greater adrenal gland weights than controls. The thickness of the motor cortex was measured in four coronal planes between $-2.33 \mathrm{~mm}$ to $-0.3 \mathrm{~mm}$ from bregma. Regions of interest that corresponded to published maps of forelimb and hind-limb representations were analyzed together. Rats in the skill-learning condition had significantly thicker medial cortical areas in the two anterior planes ( -0.8 and $-0.3 \mathrm{~mm}$ from bregma). These regions correspond to previously mapped hind-limb representations. The exercise group had greater thickness of the medial region at $-0.8 \mathrm{~mm}$ from bregma. Cortical thickness in all conditions varied significantly along the medial to lateral axis. For both treatments, the effects were restricted to medial and anterior regions of interest rather than posterior or lateral regions of interest. The results indicate that robust exercise, in addition to skill learning, is capable of altering the thickness of the motor cortex, but that the effects are restricted rather than distributed within the regions studied.
\end{abstract}

Rearing rats in an enriched environment increased brain weight (Krech et al. 1960) and visual cortical thickness (Diamond et al. 1964). The effects of enriched housing generalize across species (Floeter and Greenough 1979) and life stages (Green et al. 1983). In this environment, animals are exposed to a rich spatial environment, daily novelty, and an increased opportunity for physical activity. It is less clear which components of the enriched environment are responsible for the changes reported. The greater opportunity for physical activity cannot account for the effects entirely because the enriched environment significantly increases cortical weight, whereas exercise does not (Huntley and Newton 1972). Huntley and Newton (1972) noted that the running wheel group in their study had cortical/subcortical weight ratios that were between the ratios of the enriched and inactive groups. As a consequence, they concluded that the greater amount of physical activity in the enriched condition may have contributed to the effects of the enriched environment. We have previously shown that

${ }^{4}$ Corresponding author.

E-MAIL banderson@notes.cc.sunysb.edu; FAX (631) 632-7876. Article and publication are at http://www.learnmem.org/cgi/doi/ $10.1101 / \mathrm{lm} .43402$. motor-skill learning and exercise do not alter visual cortical thickness (Anderson et al. 1994), but it is possible that physical activity influences cortical thickness in areas other than visual cortex (e.g., sensorimotor cortex). No one has yet tested whether or not running alters motor cortical thickness.

To determine whether learning or physical activity in the enriched environment accounted for enrichment effects on the cerebellum (Floeter and Greenough 1979; Pysh and Weiss 1979; Black et al. 1990) trained rats on an obstacle course that required balance and coordination. To test for the effects of repetitive physical activity, two groups, voluntary exercise and forced exercise, were also compared with control rats housed in standard cages. Relative to the control rats, the rats required to traverse the obstacle course to learn new motor skills (acrobatic condition) had a thicker molecular layer and more synapses per neuron in the paramedian lobule of the cerebellum, which contains hind-limb and forelimb representations. The rats in the exercise condition had no change in the thickness of the molecular layer, volume of molecular layer per neuron, or synapses per neuron relative to controls, but they had a greater density of capillaries (Isaacs et al. 1992).

LEARNING \& MEMORY 9:1-9 @ 2002 by Cold Spring Harbor Laboratory Press ISSN1072-0502/02 \$5.00

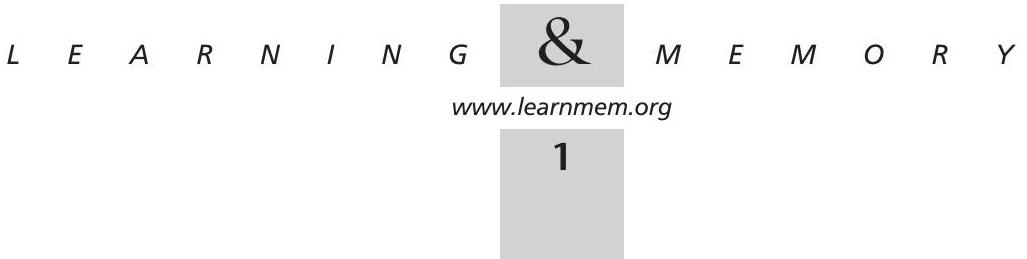


Motor-skill learning has also been shown to increase motor cortical thickness (Díaz et al. 1994), reduce motor cortical cell density (Morales et al. 1999), and increase synapses per neuron in the motor cortex (Kleim et al. 1996). Dendritic morphology is altered in forelimb representations by training rats to use a single paw to reach for small pieces of rat food (Withers and Greenough 1989). Using microstimulation mapping studies to confirm the coordinates for forelimb representations, Kleim et al. (1998) showed that similar reach training expands the size of cortical forelimb representations. More recently, it has been shown that strength training-induced alterations in cortical representation size are attributable to the skill learning and not the activity required to perform the task (Remple et al. 2001). Similarly, Plautz et al. (2000) showed that skill acquisition in monkeys altered movement representations, but a yoked repetition condition failed to alter movement representations. These studies indicate that minimal movement repetition is insufficient to induce motor cortical plasticity. No one has yet tested whether or not repetitive activity such as robust exercise can alter the structure of limb representations in the motor cortex. The present experiment was designed to test whether or not skill learning increases the thickness of the motor cortex in posterior regions of the motor cortex that have not been previously investigated.

Much of the work on plasticity in the motor cortex refers to "activity-dependent" plasticity. In support of such a concept, robust changes in neuronal electrophysiological activity have been shown to induce structural plasticity in the cortex (Keller et al. 1992; Rutledge et al. 1974). Similarly, long term potentiation (LTP) induces a change in spine density in the motor cortex (Ivanco et al. 2000). Voluntary exercise is a behavior that activates motor cortical neurons (Vissing et al. 1996; Gross et al. 1980) and increases motor cortical metabolic capacity (McCloskey et al. 2001), but it does not require the acquisition of new spatiotemporal muscle sequences or novel combinations of previously learned movements, both being distinguishing characteristics of skill learning (Sanes and Donoghue 2000). Furthermore, voluntarily exercising rats are active over more of each day than rats in yoked activity conditions commonly used to control for activity during skill learning. If plasticity in the motor cortex is "activity-dependent", then repetitive behaviors that activate the motor cortex should cause cortical plasticity. Although there have been a number of studies using activity-control conditions that yoke the distance traveled during skill learning to distance walking (Kleim et al. 1996; Kleim et al. 1998), few have assessed the effects of vigorous repetitive activity on motor cortical plasticity. The present study tests the hypothesis that motor cortical thickness will be influenced by the repetitive activity associated with voluntary wheel running.

Motor cortical thickness was measured after rats underwent three conditions: voluntary exercise (VX), the ac- robatic condition (AC) that requires motor-skill learning, or an inactive control (IC) condition. Thickness measures were taken from areas that overlap with motor cortical regions measured by Kleim et al. (1996), and more caudal areas that were reported to contain hind-limb representations (Hall and Lindholm 1974; Neafsey et al. 1986). The latter have not been tested for any form of structural plasticity after skill learning or activity. Because the boundaries of the representations cannot be easily determined in either the medial to lateral or anterior to posterior axes, we measured cortical thickness from four comparable coronal planes, through the regions of interest, and used subcortical landmarks as well as atlas coordinates to identify areas previously mapped to contain representations of forelimbs and hind limbs (see Fig. 1).

\section{RESULTS}

A one-way ANOVA was used to analyze the average number of errors in the AC group for 5-d blocks over the training period. The AC rats gradually reduced their errors over the first $26 \mathrm{~d}$ of obstacle course training $(\mathrm{F}[4,50]=8.38$, $p<.0001$; see Fig. 2A). Student Newman-Keuls post hoc analysis indicated that the acrobatic rats made significantly fewer mean errors per task on d 11-15 and 21-26 relative to the first $10 \mathrm{~d}(p<.05)$. Several tasks were particularly difficult for the rats to master. The dowel rod required the rats to modify their instinct to rapidly flip their tail in the opposite direction of their body when they lost their bal-

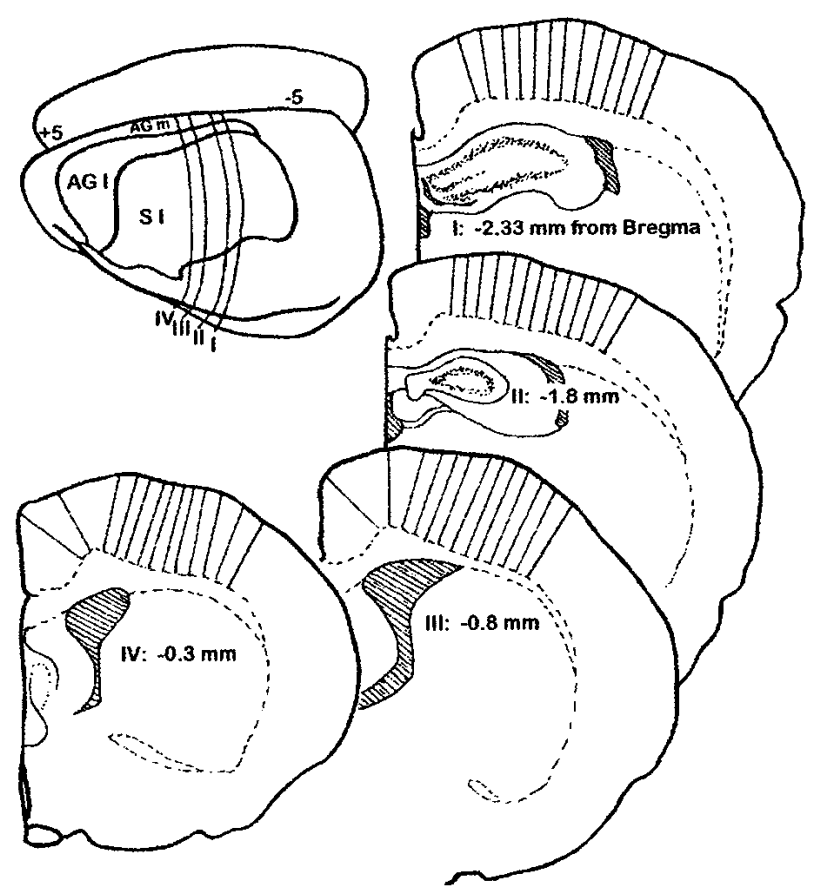

Figure 1 Schematic diagram of each of the four coronal planes investigated with the relative positions of the 13 measures of cortical thickness in each plane.

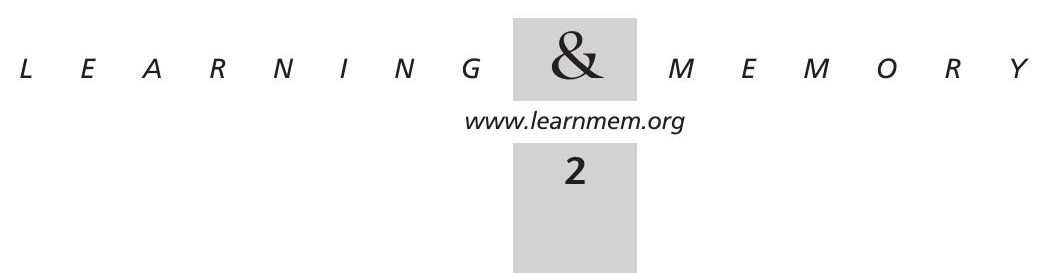




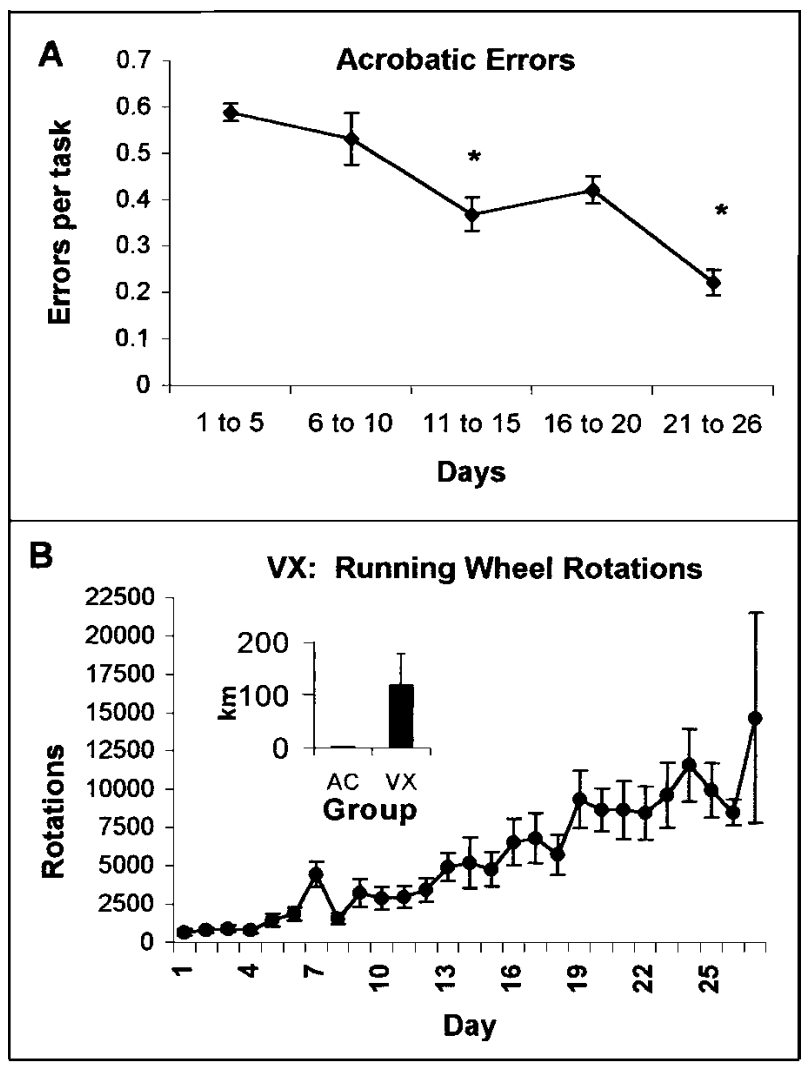

Figure 2 (A) Over the $26-29 \mathrm{~d}$ of training, the acrobatic rats made significantly fewer mean errors per task on d 11-15 and 21-26 of training relative to the first $10 \mathrm{~d}$ (see $*, p<.05$ ). (B) The rats in the voluntary exercise $(\mathrm{VX})$ group increased the number of rotations ran per day over the training period. Over the 26-29 d, the VX group traveled an average of $119 \mathrm{~km}$. The acrobatic condition (AC) group traveled a total of $0.1253 \mathrm{~km}$ over the obstacle course.

ance. The counterbalance was initially so strong that the rats pulled themselves all the way over to the tail side of the dowel and slipped completely under the dowel. With time, the distance and speed of the tail movement was reduced until it effectively provided the balance needed to correct the position of the body back to the top of the dowel rod. Similarly, the rats had great difficulty with the spring bridge, three 4-inch wide boards attached to the apparatus and each other by springs. This obstacle was introduced with tight springs that were relaxed over time until the middle board was hanging well below the rats' starting position. Over time, they learned to grasp the eye screws at the end of the boards (the appropriate diameter for their paws) and progress slowly rather than rapidly over the bridge. Some of the tasks required the rats to support their own body weight in unusual positions (e.g., primarily on the forelimbs) or to require the rats to lift their body weight or balance it onto a small space. An ANOVA (litter by group) indicated that the AC group had significantly greater heart weight than the IC group $(\mathrm{F}[1,15]=18.46, p<.025$, see Fig. 3A).
The VX rats entered the running wheels on the first day and were able to rotate the wheels rapidly. Over time the rats appeared to increase the duration and speed of running. This was seen as a significant increase in the number of daily wheel rotations over the duration of the experiment $(\mathrm{F}[4]=13.524), p<.01$, see Fig. $2 \mathrm{~B})$. The VX group had significantly greater heart weight $(F[1,15]=12.20$, $p<.025$, see Fig. 3A) and adrenal gland weight than the IC group $(\mathrm{F}[1,15]=48.40, p<.01$ see Fig. $3 \mathrm{~B})$.

Because the exercise and skill-learning conditions differed on several dimensions (e.g., time in activity per day and speed of movements) they were not directly compared. To test the hypothesis that skill learning would alter the thickness of cortical limb representations, we ran a repeated measures ANOVA using individual cortical positions as a within-subjects factor and group (AC vs. IC) and litter as

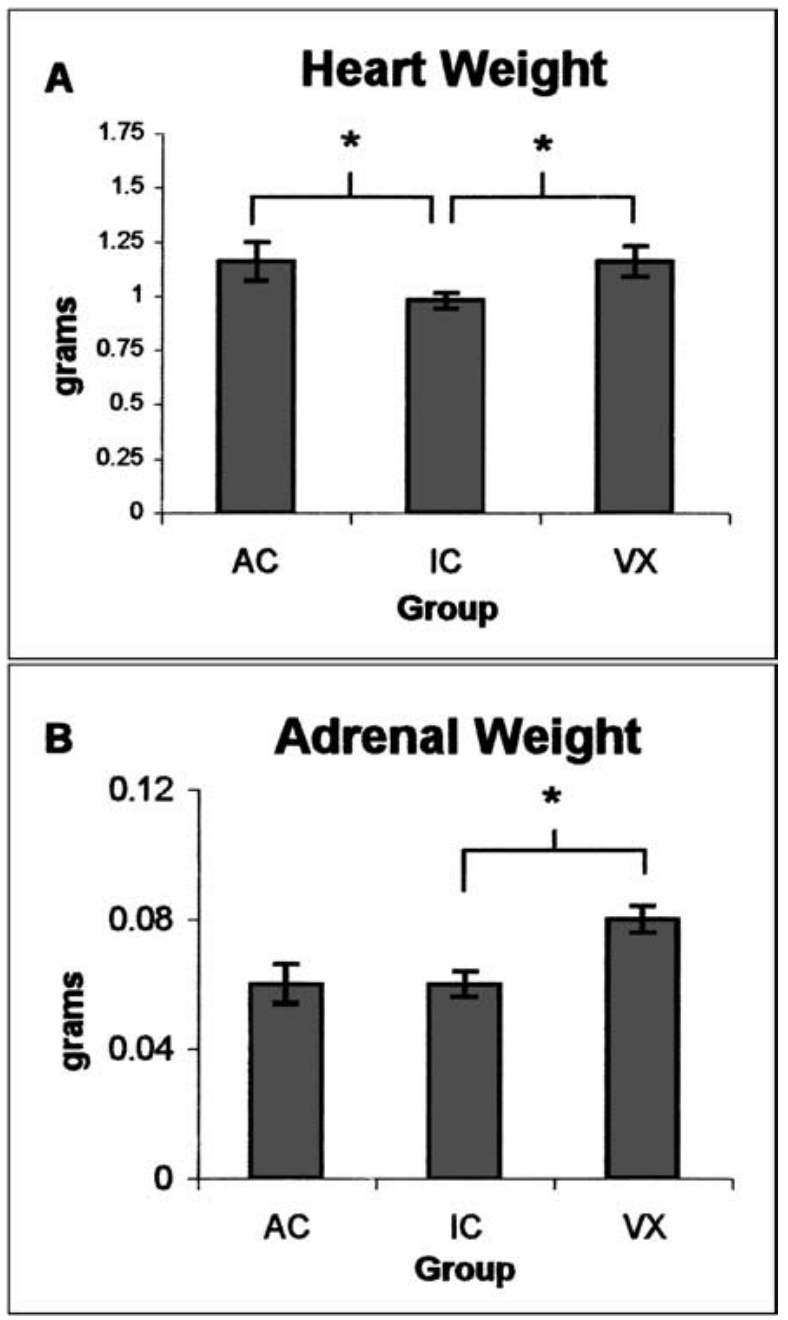

Figure 3 (A) Both the $\mathrm{AC}$ and $\mathrm{VX}$ groups had greater heart weight relative to the inactive condition (IC) group. (B) The VX group had significantly greater adrenal weight relative to the IC group. $*=p<.025$.

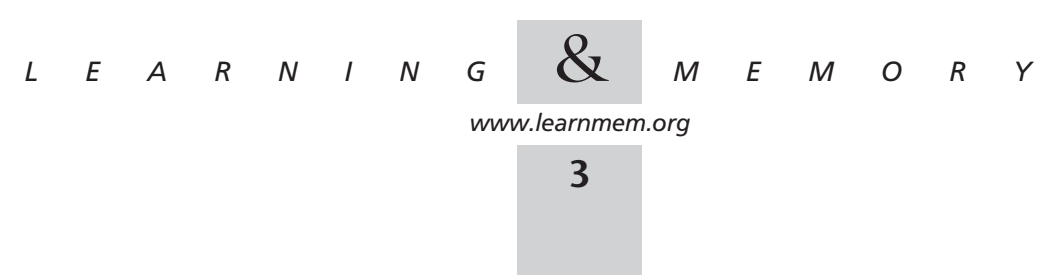


between-subjects factors. Previous maps of limb representations were used as an a priori guide to establish ROI. The following analyses were between the AC and IC conditions for each of the ROIs in each of the four coronal planes investigated. At $-2.33 \mathrm{~mm}$ from bregma, the thickness for both the AC and IC groups changed significantly across positions in the posterior ROI $(\mathrm{F}[4,12]=9.77, p<.001)$ with an interaction between group and cortical position $(\mathrm{F}[4,6]=5.72, p<.01$; see Fig. $4 \mathrm{~A})$ but no main effect of group. At $-1.8 \mathrm{~mm}$ from bregma, there was a significant effect of position $(\mathrm{F}[7,12)=69.41, p<.001$, see Fig. 4B) but no effect of group in the posterior ROI. The repeated measures analysis for the medial ROI measured at $-0.8 \mathrm{~mm}$ posterior to bregma revealed an effect of group $(\mathrm{F}[1,18]=22.35, p<.01)$ and position $(\mathrm{F}[2,12]=12.21$, $p<.01$ ) but no interactions (see Fig. $4 \mathrm{C}$ ). In the medial ROI at $-0.3 \mathrm{~mm}$ posterior to bregma there was a main effect of group $(\mathrm{F}[1,36]=89.46, p<.001)$, litter $(\mathrm{F}[5,4]=43.02$, $p<.01)$, and a group by litter interaction $(\mathrm{F}[5,2]=13.54$, $p<.025$; see Fig. 4D). Only sections at -0.8 and $-0.3 \mathrm{~mm}$ posterior to bregma contained lateral ROIs. At $-0.8 \mathrm{~mm}$ from bregma, the analysis for the AC and IC conditions revealed a trend for an effect of group $(F[1,18]=11.81$, $p=.026)$ and an effect of position $(\mathrm{F}[2,12]=21.16$, $p<.001$; see Fig. $4 \mathrm{C}$ ). In the lateral ROI at $-0.3 \mathrm{~mm}$ posterior to bregma there was a trend for an effect of group $(\mathrm{F}[1,36]=8.95, p=.043)$ and a trend for an effect of position $(\mathrm{F}[5,12]=2.92, p=.039$; see Fig. 4D). The site of effects between the AC and IC groups corresponded to positions that lie over atlas coordinates $1.35 \mathrm{ML} / 2.53 \mathrm{DV}$ and $2.11 \mathrm{ML} / 2.8 \mathrm{DV}$ at $-0.8 \mathrm{~mm}$ from bregma. Likewise, the site between 1.8 ML/2.47DV and 2.24ML/2.6 DV at $-0.3 \mathrm{~mm}$ from bregma.

To test the hypothesis that robust exercise would alter the thickness of M1 posterior to bregma, we ran a repeated measures ANOVA using individual cortical positions within ROIs as the withinsubjects factor and group and litter as the between-subjects factors. At -2.33 $\mathrm{mm}$ posterior to bregma, there were no main effects or interactions for the VX and IC groups (see Fig. 5A). In the posterior ROI at $-1.80 \mathrm{~mm}$ posterior to bregma, there were no main effects of group $(F[1,48]=7.45$, $p=.0525)$ but a main effect of position $(\mathrm{F}[7,6]=19.90, p<.0001$; see Fig. $5 \mathrm{~B})$. In the medial ROI at $-0.8 \mathrm{~mm}$ posterior to bregma, the VX versus IC comparison revealed a main effect of group $(\mathrm{F}[1,18]=14.00, \quad p<.025)$ and an effect of position $(\mathrm{F}[2,12]=15.20$, $p<.01$; see Fig. $5 \mathrm{C}$ ). The atlas coordinates for the anterior medial site with a significant group effect are cortical positions that lie above white matter coordinates between the borders of $1.35 \mathrm{ML} / 2.53 \mathrm{DV}$ and $2.11 \mathrm{ML} / 2.8 \mathrm{DV}$. For the VX versus IC comparison of the medial ROI at $-0.30 \mathrm{~mm}$ posterior to bregma there was a trend for an effect of group $(\mathrm{F}[1,12]=11.62, p=.027$; see Fig. 5D) but no main effect of position or interactions. In the lateral ROI in the coronal plane at $-0.8 \mathrm{~mm}$ posterior to bregma, there was no main effect of group, or interactions, but there was an effect of position $(\mathrm{F}[2,12]=20.15, \quad p<.001 ;$ see $\mathrm{Fig}$. 5C). In the lateral ROI in the coronal plane at $-0.3 \mathrm{~mm}$ posterior to bregma,

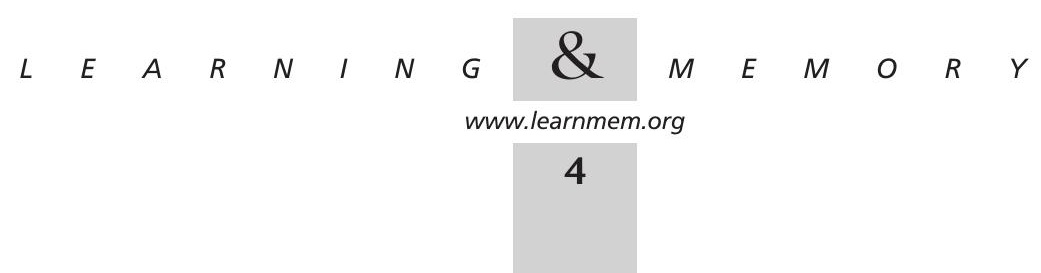




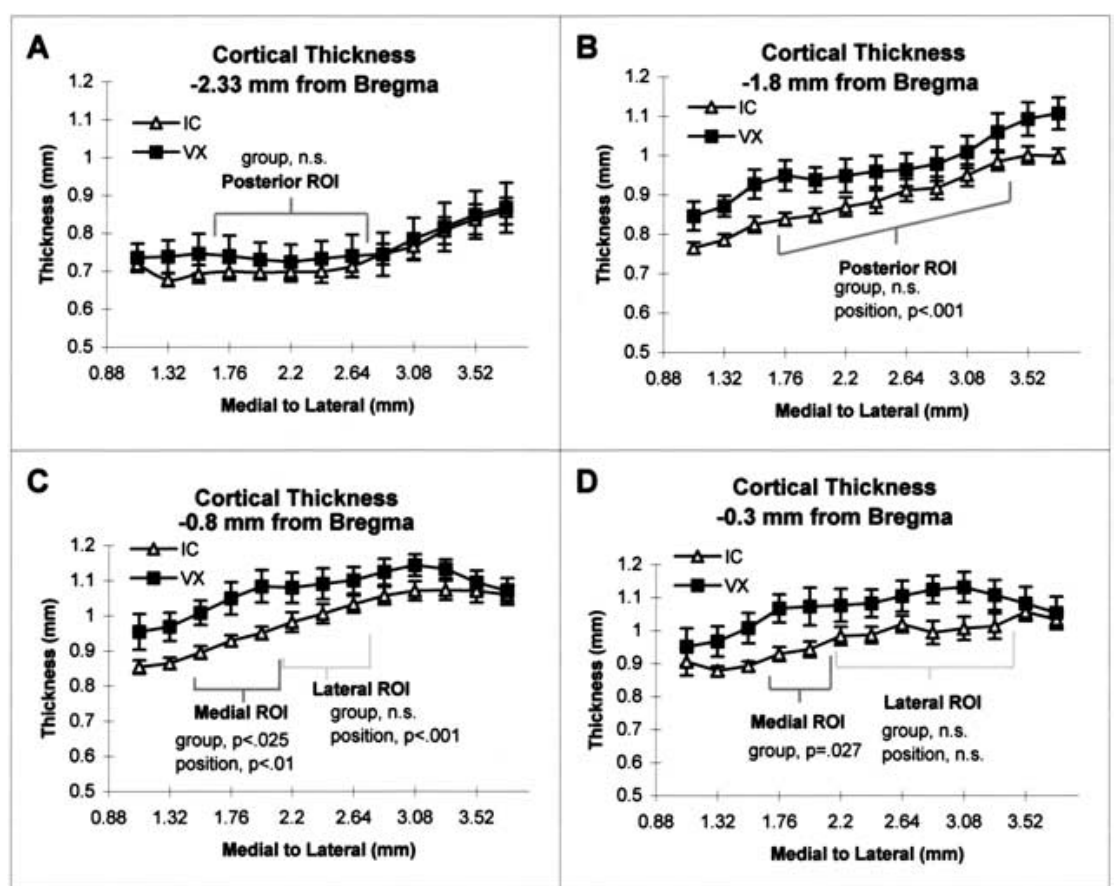

Figure 5 (A) Comparison of the VX group with the IC group: At the coronal plane equivalent to $-2.3 \mathrm{~mm}$ from bregma as seen in Zilles (1985), there was no significant difference between the VX and IC groups in the ROI. There was no effect of litter, position, and no interactions. (B) At the coronal plane equivalent to $-1.8 \mathrm{~mm}$ from bregma (Zilles 1985), there was no effect of group and no interactions, but thickness varied by position over the ROI $(p<.001)$. (C) At the coronal plane equivalent to that of $-0.8 \mathrm{~mm}$ from bregma (Zilles 1985), the thickness of the medial ROI was greater in the VX group than the IC group $(p<.025)$. Exercise did not alter the thickness of the lateral ROI. In both ROls, thickness varied over position $(p<.01)$. (D) At the coronal plane equivalent to that of $-0.3 \mathrm{~mm}$ from bregma (Zilles 1985), there was a trend for the VX group to have thicker cortices at the medial ROI than the IC group $(p=.027)$. Exercise did not alter the thickness of the lateral ROI. There was no effect of position in either ROI.

there were no main effects within subjects effects or interactions (see Fig. 5D).

All 13 positions were also analyzed for each plane of section. The AC group had significantly thicker cortex in the planes $-0.30 \mathrm{~mm}$ from bregma $(\mathrm{F}[1,15]=12.21$, $p=.025)$. There was a trend for the AC group to have overall greater thickness at $-0.8 \mathrm{~mm}$ from bregma $(\mathrm{F}[1,15]=9.20, p=.039)$. There was no effect of group at either -1.8 or $-2.33 \mathrm{~mm}$ posterior to bregma. The exercise group had a trend for thicker cortices across the 13 measures at $-1.8 \mathrm{~mm}$ from bregma $(\mathrm{F}[1,15]=9.21, p<.039)$ but not in any other plane measured. An effect of position was significant in all four coronal planes $(p<.001)$ for all comparisons.

\section{DISCUSSION}

Rats in the AC group required only 5-10 min per day on the obstacle course. Despite the short time of training, this group had greater heart weight relative to rats in the control group. This finding indicates that there was an aerobic component to training despite the slow pace and short time on the tasks. The strenuous nature of some of the tasks might account for the greater metabolic demand that was likely related to the increase in heart weight. For example, the spring bridge required the rats to carefully shift their body weight onto forelimbs while maintaining the position of the boards in space. The same tasks, however, required the rats to learn new spatiotemporal muscle activation patterns, which Sanes and Donoghue (2000) suggest is a behavioral distinction of motor-skill learning (see qualitative descriptions in the Results section). The rats in the AC group reduced the number of errors as training progressed despite the increasing difficulty of the tasks. These findings support the conclusion that the AC group acquired new motor skills.

When all 13 positions in each plane were analyzed together, the AC condition caused a significant increase in thickness only at $-0.3 \mathrm{~mm}$ from bregma. When cortical thickness was analyzed by ROI, the AC condition significantly affected only the medial regions of interest in the two most anterior coronal planes that were investigated, -0.8 and $-0.3 \mathrm{~mm}$ from bregma. The medial ROI corresponds to hindlimb representations from cytoarchitectonic maps (Zilles 1985) and from microstimulation mapping studies (Neafsey et al. 1986; Hall and Lindholm 1974). We found only a trend for increased thickness in the anterior lateral ROIs, which correspond to cytoarchitectonically mapped forelimb representations of Zilles (1985). These regions also correspond to forelimb somatosensory representations mapped by Hall and Lindholm (1974) and to some individual representations but not to the averaged forelimb movement representations described by Neafsey et al. (1986). It is possible that the lateral ROI at $-0.8 \mathrm{~mm}$ from bregma reflects additional hindlimb representations, but that the failure to find a significant effect reflects greater variance because of individual differences in the lateral border.

The altered morphology in the anterior medial ROIs is most likely related to the skill learning required rather than the somatomotor activity in this condition. After all, Kleim et al. (1996) have shown that the AC condition alters neuronal density and synaptic numbers per neuron in M1 but failed to show that movement yoked for the distance covered by the AC group influences these variables. That study included regions of M1 that were anterior and posterior to

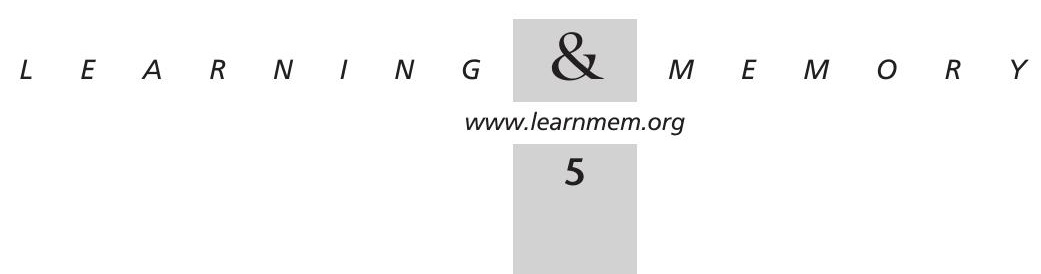


bregma. Their posterior regions overlap with the two most anterior coronal planes investigated in the present study. Thus the results in the AC group seem unlikely to be attributed solely to the movement associated with performing the task in a 2-15-min period each day unless they are related to differences in the strength required for the movements. Based on Kleim et al. (1996), we suspect that the thicker anterior medial ROI in the AC groups reflects, in part, an increase in the number of synapses per neuron. This interpretation is consistent with findings that the enriched environment can increase cortical thickness (Diamond et al. 1966) and that changes in thickness correspond to reductions in neuronal density and increases in the number of synapses per neuron (Turner and Greenough 1985) in the visual cortex. Similarly, in the cerebellum, the AC condition increased molecular layer volume and the number of synapses per neuron (Black et al. 1990).

The VX rats had constant access to their wheels. Over the training period, they increased the number of daily wheel rotations. Heart and adrenal gland weight were significantly greater in this group than in the controls. The greater heart weight relative to the IC condition likely reflects the aerobic conditioning component of this treatment. Similarly, the increase in adrenal weight is consistent with the robust level of activity the VX rats pursued in the running wheel. This finding is consistent with previous reports of adrenal gland hypertrophy after treadmill training (Schmidt et al. 1992) and voluntary activity for 3 mo in mice (Samorajski et al. 1987). Adrenal hypertrophy is not necessarily a predictor of the direction of exercise-related changes in glucocorticoid secretion (Tharp 1975) but may indicate a greater capacity for epinephrine secretion (Kjaer 1998). The peripheral effects of exercise leave open the possibility that exercise indirectly influences the brain through hormonal or metabolic factors. Such indirect effects might be expected to cause broadly distributed effects in the central nervous system (CNS) rather than effects restricted to structures related to movement or sensory feedback from movement.

Running increased cortical thickness only in the anterior medial ROI at $-0.8 \mathrm{~mm}$ posterior to bregma, with no significant effect on the anterior lateral ROI or the posterior ROIs. The restricted region of the exercise effect indicates that the plasticity is not related to general hormonal or metabolic effects of exercise but instead is a likely outcome of neuronal activity related to the sensation and/or production of the repetitive movements. The finding that vigorous repetitive activity can alter motor cortical thickness, although in a restricted region, is consistent with the concept of activity-dependent plasticity in the motor cortex. Given that yoked activity control conditions do not produce motor cortical plasticity (Kleim et al. 1996; Morales et al. 1999), it seems reasonable to hypothesize that activity-dependent plasticity resulting in changes in cortical thickness are limited to repetitive movements that take place repeatedly over the course of a day, rather than short-duration, moderate movements such as those yoked to skill learning.

The hypothesis that robust repetitive movements cause use- (or activity-) dependent adaptation is consistent with findings that increased neuronal activity from electrical stimulation is sufficient to induce morphological change in the cortex (Rutledge et al. 1974; Keller et al. 1992). Based on a description of motor learning by Sanes and Donoghue (2000), the exercise-induced plasticity is a form of motor learning categorized more specifically as motor adaptation. Motor adaptation is associated with the repetition of already learned movements. This is distinct from skill learning, which has been proposed to require the acquisition of new spatiotemporal muscle activation patterns. Consistent with the concept of motor adaptation, the $\mathrm{VX}$ rats enter the wheel on the first day and begin running. The rats do not acquire new spatiotemporal muscle activation sequences. The repetition of running might bring about sensorimotor adaptation that involves numerous forms of plasticity that are directed toward increasing endurance. Some forms of plasticity related to sensorimotor adaptation would come about in the periphery (e.g., adaptation in striate muscles and the heart) and some centrally. The latter might include greater density of capillaries, which has been reported in the cerebellum, but this form of plasticity does not require an increase in tissue volume in the cerebellum (Isaacs et al. 1992). Likewise, endurance could be increased by changing the capacity for oxidative metabolism, which has been shown in the motor cortex after 6 mo of voluntary exercise (McCloskey et al. 2001), but it is doubtful that this form of plasticity would require an increase in tissue volume unless there is a substantial increase in mitochondrial volume. Adding connections that can alternate responsibility for locomotion in the wheel could also be a mechanism for increasing endurance that would require an increase in cortical thickness. It is also possible that refining movements through the inhibition of unnecessary contractions might be a strategy to reduce the energy demands associated with running. These latter forms of adaptation might also require a change in the number of synapses per neuron with the functional outcome being similar to motor-skill learning.

Both skill learning and exercise failed to affect the most posterior ROIs. Significant differences were restricted to one or both anterior medial ROIs for the two treatment groups. The differential pattern of effects from the anterior to posterior planes and medial to lateral regions of interest indicates that neither condition caused a generalized effect on cortical thickness, but that both influenced select areas. Further support for the specificity of the effects comes from previous findings that the $\mathrm{AC}$ and $\mathrm{VX}$ conditions failed to influence the thickness of the visual cortex but caused structural plasticity in the cerebellum in an earlier study (Anderson et al. 1994). The restricted nature of the effects

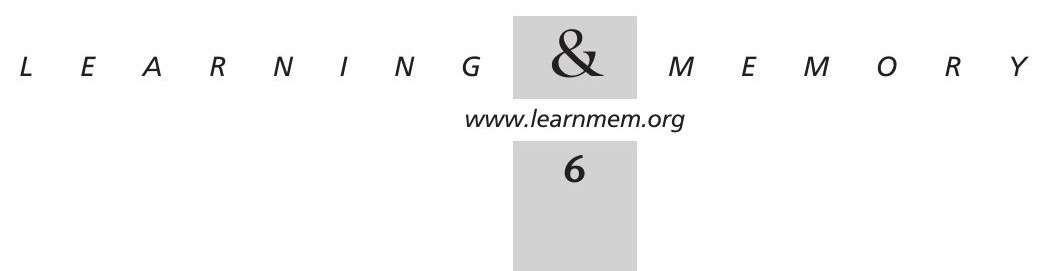


in the VX condition corresponds to our previous findings that exercise-related effects on CNS metabolic capacity are also regionally selective rather than broadly distributed (McCloskey et al. 2001). These findings further support the hypothesis that exercise-related plasticity in the CNS is specifically related to neuronal activity associated with the movements and not a consequence of the general hormonal or cardiovascular effects of exercise.

The present results differ from the effects of these same treatment conditions on the cerebellum (Black et al. 1990; Isaacs et al. 1992). In that region, the AC condition caused an increase in the number of synapses per neuron and in tissue volume per neuron relative to the control group, but the VX group did not differ from controls on these measures. The VX condition caused greater capillary density relative to the IC group (Isaacs et al. 1992). The 6-mo-old female VX group in the present study ran six times the number of rotations than the 10-mo-old females in the previous study (Black et al. 1990), despite having identical running wheels. Accordingly, adrenal gland weight increased in the present VX group relative to the IC group but not in 10-mo-old exercising rats (Isaacs et al. 1992). It remains to be seen whether this difference in the pattern of findings reflects differences in how these two motor regions respond to exercise or differences in the intensity of the exercise across the studies.

In the present study, we chose to measure individual positions and use the repeated measures analysis because previous experience led us to believe there is substantial variability in thickness across the motor cortex. The present study confirmed the existence of significant variability over the medial to lateral measures in each of the four coronal planes, with the thickness increasing as distance from midline increased. This variability has implications for future studies using representative random sampling for measures that rely in part on cortical thickness.

In summary, the AC condition decreased the number of errors on the obstacle course over training, indicating that they acquired the skills needed to traverse the obstacle course in the 2-15-min daily training period. The AC group had greater heart weight and increased cortical thickness in the medial regions of interest in both anterior planes. It seems reasonable to hypothesize that for the AC condition, the differences in thickness correspond to differences in the number of synapses per neuron. The VX group increased the number of rotations over the training period. Relative to the IC group, the VX group had greater heart and adrenal gland weight and cortical thickness in the medial ROI at $-0.8 \mathrm{~mm}$ from bregma. This is the first study to indicate that exercise can alter cortical thickness. These effects on cortical thickness were restricted to one anterior medial ROI. Thus, the effects appear to be related specifically to neuronal activity associated with the robust repetitive movements rather than general, distributed effects of exercise in the periphery. Future studies will be needed to determine whether the plasticity that underlies the need to increase cortical volume is at the cellular and/or ultrastructural level and whether it is similar or different from the changes in the number of synapses per neuron that previously have been shown to occur with skill learning (Kleim et al. 1996; Kleim et al. 1998).

\section{MATERIALS AND METHODS}

\section{Subjects}

Twenty-three female Long-Evans hooded rats, consisting of six sets of littermates, were reared in groups of three to four per standard laboratory cage. At 6 mo of age the littermates were equally divided across three experimental groups. These were divided so that there were seven rats in the two treatment conditions and nine rats in the control condition. In each group there were one to two rats from each of the six littermate sets.

\section{Behavioral Conditions}

The seven rats in the AC traversed an elevated series of platforms connected by various obstacles consisting of a 4-inch wide board, 1 1/2-inch wide board, 3/4-inch wide board, rope and spring bridges, dowels, braided rope, chain, horizontal and vertical ladders, weather stripping, and various seesaws, each with an average length of $70 \mathrm{~cm}$. Each day or two, a new and more difficult task was added for all rats. For example, the rats started by walking over wide boards and progressed over days to narrower boards until they traversed dowel rods. Observers recorded the number and types of errors, which included loss of balance, misplacement of a paw, and slipping under a task by hanging on upside down and grooming. Grooming indicated task avoidance. The course required a considerable amount of motor-skill learning. Rats traversed the obstacle course for five trials a day over a period ranging from 2 to $15 \mathrm{~min}$.

The seven rats in the VX group were housed individually with free access to a running wheel $(34 \mathrm{~cm} \times 10.5 \mathrm{~cm})$ attached to the home cage. Nine rats in the IC were housed in individual cages. These rats had minimal opportunity for either motor learning or physical exercise. The rats in the inactive condition and voluntary exercise condition were handled daily for $3 \mathrm{~min}$ each.

\section{Histology}

After training for 26-29 d, the rats were anesthetized with sodium pentobarbital $(75 \mathrm{mg} / \mathrm{kg})$ and intracardially perfused with Tyrode's solution followed by $2 \%$ paraformaldehyde $/ 2.5 \%$ glutaraldehyde fixative in $0.1 \mathrm{M}$ phosphate buffer. Wet weights of hearts and adrenal glands were also collected. Brains were embedded in $10.6 \%$ celloidin, sectioned at $30 \mu \mathrm{m}$ thick, stained with Methylene BlueAzure II, and traced at 22.5x. Cytoarchitectonic boundaries of motor cortex are difficult to establish, particularly in the anterior to posterior axis. Furthermore, these boundaries are more difficult to apply to forelimb and hind-limb representations. Therefore, we used previously published descriptions of the cytoarchitectonic locations of limb representations (Zilles 1985) that overlap with hind-limb and posterior forelimb representations identified through microstimulation mapping (Neafsey et al. 1986). Because of the variability in the thickness of the motor cortex, we measured thickness of cortical layers I-VI in four homologous coronal planes equivalent to the coronal planes at $-2.33 \mathrm{~mm},-1.8,-0.8$, and -0.3 $\mathrm{mm}$ from bregma (Zilles 1985). Voluntary running has previously been shown to alter the capacity for oxidative metabolism in motor

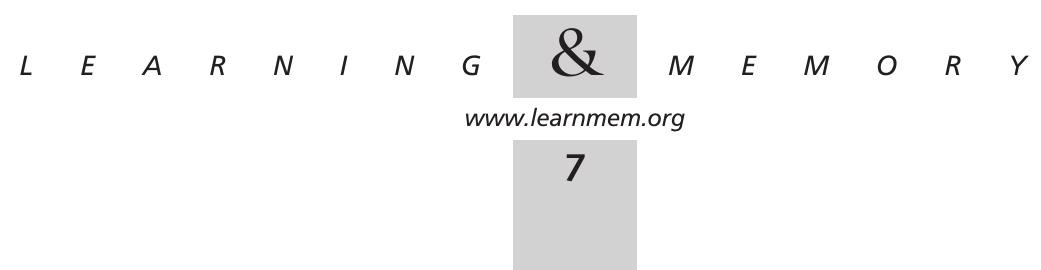


cortical areas within these planes (McCloskey et al. 2001). Within each plane, 13 thickness measures were taken every $0.222 \mathrm{~mm}$ along the white matter starting at $1.1 \mathrm{~mm}$ lateral from midline. A line perpendicular to the white matter was drawn through the six layers of cortex, and the distance between the pia matter and white matter was measured (see Fig. 1). No attempt was made to correct for tissue shrinkage, which was expected to be equivalent across treatment conditions. Although limb representations, particularly hind-limb representations, vary from rat to rat, choosing stereotaxic coordinates of previously mapped limb representations to decide a priori the coordinates for analyses is preferable to viewing the data and performing post hoc analyses. The latter method greatly increases the probability of making a type I error (Howell 2001). As a result, we used the previously mapped positions as a guide for grouping positions for analyses. The presumed hind-limb representations in the coronal plane of $-2.33 \mathrm{~mm}$ from bregma corresponded to cortex lying between perpendicular lines drawn from the white matter at atlas coordinates 1.55 medial to lateral axis (ML) and 2.35 dorsal to ventral axis (DV) and a point equal to $2.89 \mathrm{ML}$ and $2.78 \mathrm{DV}$, all relative to bregma as shown in Zilles (1985). This position is one of two regions of interest (ROIs) that will be referred to as a posterior ROI. The presumed hind-limb representations in the coronal plane of $-1.80 \mathrm{~mm}$ from bregma were between perpendicular lines drawn from the white matter at atlas coordinates 1.44 ML and 2.37 DV and from 3.02 ML and 2.97 DV, all relative to bregma as shown in Zilles (1985). This was the second posterior ROI. The presumed hind-limb representations in the coronal plane of $-0.80 \mathrm{~mm}$ from bregma were between perpendicular lines drawn from the white matter at atlas coordinates $1.35 \mathrm{ML}$ and $2.53 \mathrm{DV}$ and from 2.11 ML and 2.80 DV, all relative to bregma as shown in Zilles (1985). This will be referred to as an anterior medial ROI. The lateral border for the presumed hind-limb representation was also used as the medial border for the forelimb representations, whereas the lateral border started at atlas coordinates $2.81 \mathrm{ML}$ and 3.00 DV. This will be referred to as one of the anterior lateral ROIs. The presumed hind-limb representations in the coronal plane of $-0.30 \mathrm{~mm}$ from bregma were between perpendicular lines drawn from the white matter at 1.80 ML and $2.47 \mathrm{DV}$ and from $2.24 \mathrm{ML}$ and 2.60 DV. This was the second of two anterior medial ROIs. The lateral border for the presumed hind-limb representation was also used as the medial border for the forelimb representations, whereas the lateral border started at $3.54 \mathrm{ML}$ and $3.12 \mathrm{DV}$. This was the second of two anterior lateral ROIs. Every position in each plane was measured in both hemispheres and averaged. Each position is reported as the relative distance in the experimental tissue. Because celloidin embedding causes shrinkage that leaves the coronal section flatter and wider than frozen sections, corresponding positions were determined by calculating the proportional distance along the white matter. Averages for each position were analyzed with a repeated measures ANOVA with cortical position within a presumed representation (forelimb or hind limb) as the withinsubjects factor and group and litter as the between-subjects factors. The Bonferroni correction factor for multiple comparisons was used so that the final $\alpha$ level was .05, with an $\alpha$ level of .025 for each comparison.

\section{ACKNOWLEDGMENTS}

We thank Chris Wallace for helping us build the obstacle course and Franz Davis and Kimberly Goldberg for help with the behavioral manipulations. We also thank Karl Haglund and William Arloff for help measuring the cerebral cortex.
The publication costs of this article were defrayed in part by payment of page charges. This article must therefore be hereby marked "advertisement" in accordance with 18 USC section 1734 solely to indicate this fact.

\section{REFERENCES}

Anderson, B., Li, X., Alcantara, A., Isaacs, K., Black, J., and Greenough, W.T. 1994. Glial hypertrophy is associated with synaptogenesis following motor-skill learning, but not with angiogenesis following exercise. Glia 11: 73-80.

Black, J.E., Isaacs, K.R., Anderson, B.J., Alcantara, A.A., and Greenough, W.T. 1990. Learning causes synaptogenesis, whereas motor activity causes angiogenesis, in cerebellar cortex of adult rats. Proc. Natl. Acad. Sci. 87: 5568-5572.

Diamond, M.C., Krech, D., and Rosenzweig, M.R. 1964. The effects of an enriched environment on the histology of the rat cerebral cortex. $J$. Comp. Neurol. 123: 111-120.

Diamond, M.C., Law, F., Rhodes, H., Lindner, B., Rosenzweig, M.R., Krech, D., and Bennett, E. L. 1966. Increases in cortical depth and glia numbers in rats subjected to enriched environment. J. Comp. Neurol. 128: $117-126$

Díaz, E., Pinto-Hamuy, T., and Fernandez, V. 1994. Interhemispheric structural asymmetry induced by a lateralized reaching task in the rat motor cortex. Eur. J. Neurosci. 6: 1235-1238.

Floeter, M.K. and Greenough, W.T. 1979. Cerebellar plasticity: Modification of Purkinje cell structure by differential rearing in monkeys. Science 206: 227-229.

Green, E.J., Greenough, W.T., and Schlumpf B.E. 1983. Effects of complex or isolated environments on cortical dendrites of middle-aged rats. Brain Res. 264: 233-240.

Gross, P.M., Marcus, M.L., and Heistad, D.D. 1980. Regional distribution of cerebral blood flow during exercise in dogs. J. Appl. Physiol. 48: 213-217.

Hall, R.D. and Lindholm, E.P. 1974. Organization of the motor and somatosensory neocortex in the albino rat. Brain Res. 66: 23-38.

Howell, D.C. 2002 Statistical methods for psychology. pp. 372. Duxbury, Pacific Grove, CA

Huntley, M.J. and Newton, J.M. 1972. Effects of environmental complexity and locomotor activity on brain change in the rat. Physiol. Behav. 8: 725-727.

Isaacs, K.R., Anderson, B.J., Alcantara, A.A., Black, J.E, and Greenough, W.T. 1992. Exercise and the brain: Angiogenesis in the adult rat cerebellum after physical activity and motor skill learning. J. Cereb. Blood Flow Metab. 12: 110-119.

Ivanco, T.L., Racine, R.J., and Kolb, B. 2000. Morphology of layer III pyramidal neurons is altered following induction of LTP in sensorimotor cortex of the freely moving rat. Synapse 37: 16-22.

Keller, A., Arissian, K., and Asanuma, H. 1992. Synaptic proliferation in the motor cortex of adult cats after long-term thalamic stimulation. $J$. Neurophysiol. 68: 295-308.

Kjaer, M. 1998. Adrenal medulla and exercise training. Eur. J. Appl. Physiol. Occup. Physiol. 77: 195-199.

Kleim, J.A., Bombay, S., and Nudo, R.J. 1998. Functional reorganization of the rat motor cortex following motor skill learning. J. Neurophysiol. 80: 3321-3325.

Kleim, J.A., Lussnig, E., Schwarz, E.R., Comery, T.A., and Greenough, W.T. 1996. Synaptogenesis and Fos expression in the motor cortex of the adult rat after motor skill learning. J. Neurosci. 16: 4529-4535.

Krech, D., Rosenzweig, M.R., and Bennett, E.L. 1960. Effects of environmental complexity and training on brain chemistry. J. Comp. Physiol. Psychol. 53: 509-519.

McCloskey, D.P., Adamo, D., and Anderson, B.J. 2001. Exercise increases metabolic capacity in the motor cortex and striatum, but not in the hippocampus. Brain Res. 891: 1-2; 168-175.

Morales, P., Pinto-Hamuy, T. Fernández, V., and Díaz, E. 1999. Persistent neuronal density changes related to the establishment of a motor memory. Behav. Brain Res. 99: 115-121.

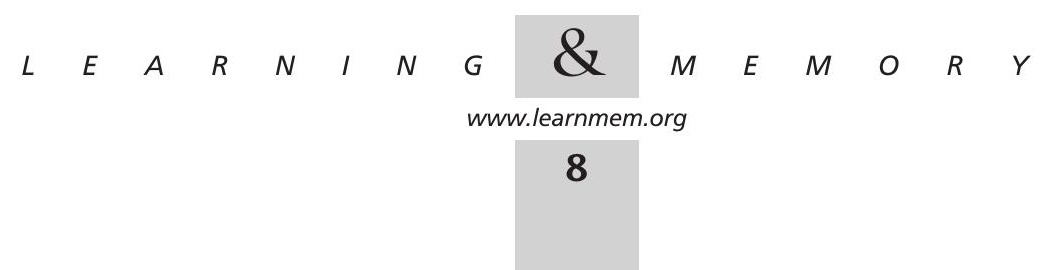


Neafsey, E.J., Bold, E.L., Haas, G., Hurley-Gius, K.M., Quirk, G., Sievert, C.F., and Terreberry, R.R. 1986. The organization of the rat motor cortex: A microstimulation mapping study. Brain Res. Rev. 11: 77-96.

Plautz, E.J., Milliken, G.W., and Nudo, R.J. 2000. Effects of repetitive motor training on movement representations in adult squirrel monkeys: Role of use versus learning. Neurobiol. Learn. Mem. 74: 27-55.

Pysh, J.J. and Weiss, G.M. 1979. Exercise during development induces an increase in Purkinje cell dendritic tree size. Science 206: 230-231.

Remple, M.S., Bruneau, R.M., VandenBerg, P.M., Goertzen, C., and Kleim, J.A. 2001. Sensitivity of cortical movement representations to motor experience: Evidence that skill learning but not strength training induces cortical reorganization. Behav. Brain Res. 123: 133-141.

Rutledge, L.T., Wright, C., and Duncan, J. 1974. Morphological changes in pyramidal cells of mammalian neocortex associated with increased use. Exp. Neurol. 44: 209-228.

Samorajski, T. Rolsten, C., Przykorska, A., and Davis, C.M. 1987. Voluntary wheel running exercise and monoamine levels in brain, heart and adrenal glands in aging mice. Exp. Gerontol. 22: 421-431.

Sanes, J.N. and Donoghue, J.P. 2000. Plasticity and primary motor vortex. Annu. Rev. Neurosci. 23: 393-415.
Schmidt, K.N., Gosselin, L.E., and Stanley, W.C. 1992. Endurance exercise training causes adrenal medullary hypertrophy in young and old Fischer 344 rats. Horm. Metab. Res. 24: 511-515.

Tharp GD. 1975. The role of glucocorticoids in exercise. Med. Sci. Sports Exerc. 7: 6-11.

Turner, A.M. and Greenough, W.T. 1985. Differential rearing effects on rat visual cortex synapses. I. Synaptic and neuronal density and synapses per neuron. Brain Res. 329: 195-203.

Vissing, J., Andersen, M., and Diemer, N.H. 1996. Exercise-induced changes in local cerebral glucose utilization in the rat. J. Cereb. Blood Flow Metab. 16: 729-736.

Withers, G.S. and Greenough, W.T. 1989. Reach training selectively alters dendritic branching in subpopulations of layer II-III pyramidals in rat motor-somatosensory forelimb cortex. Neuropsychologia 27: 61-69.

Zilles, K. 1985. The cortex of the rat: A stereotaxic atlas. Springer-Verlag, New York, NY.

Received July 23, 2001; accepted in revised form February 15, 2002.

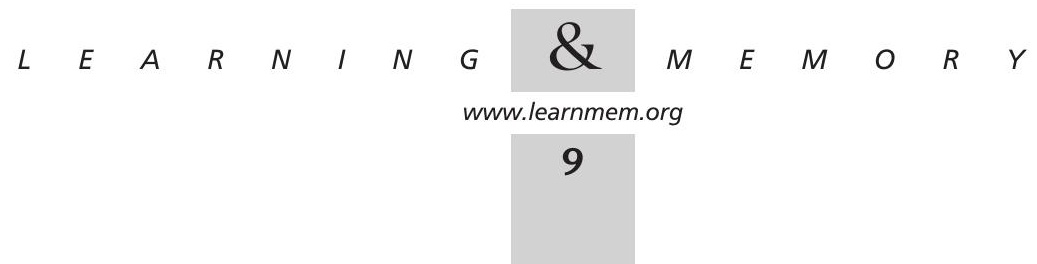




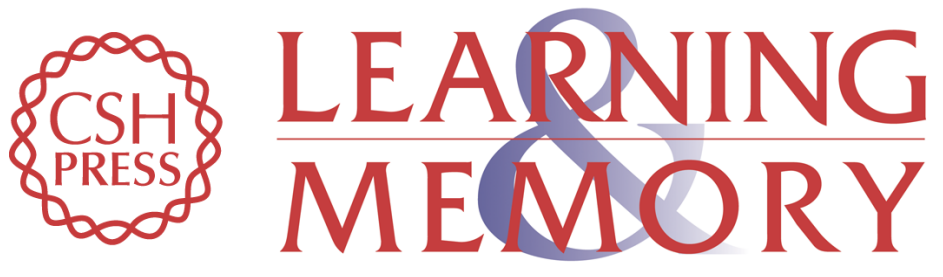

\section{Alterations in the Thickness of Motor Cortical Subregions After Motor-Skill Learning and Exercise}

Brenda J. Anderson, Paul B. Eckburg and Karen I. Relucio

Learn. Mem. 2002, 9:

Access the most recent version at doi:10.1101//m.43402

References This article cites 29 articles, 4 of which can be accessed free at:

http://learnmem.cshlp.org/content/9/1/1.full.html\#ref-list-1

License

Email Alerting Receive free email alerts when new articles cite this article - sign up in the box at the Service top right corner of the article or click here. 